\title{
Buchbesprechungen - Book Reviews
}

Gynäkologische Zytodiagnostik

H. J. Soost, S. Bauer

Thieme, Stuttgart, 1990

390 S, 482 Abb., 43 Tab., geb., DM 290-

ISBN 3-13-403105-1

Die gynäkologische Zytodiagnostik von H. J. Soost und S. Bauer ist, seit vor 35 Jahren die erste Auflage dieses Buches erschien, zum Standardwerk der gynäkologischen Zytodiagnostik geworden. Nachdem die vierte Auflage über 10 Jahre zurückliegt, war es notwendig, das Buch völlig zu überarbei-ten. Neu hinzugekommen sind Abschnitte über die Genese des Zervixkarzinoms unter besonderer Berücksichtigung der HPV-Infektionen, über die Histologie und Zytologie der Kondylome und über die Vulvazytologie. Die Nomenklatur und Befundwiedergabe in der gynäkologischen Zytologie, die von der Deutschen Gesellschaft für Zytologie im Dezember 1989 als Münchner Nomenklatur II beschlossen wurde, wird ausführlich dargestellt. Neu sind Kapitel über Materialgewin-nung, Fixierung und Versand und die Abschnitte über die Qualitätskontrolle in der Zytodiagnostik. Die Qualität der farbigen Abbildungen der zytologischen Präparate ist über jede Diskussion erhabeń und empfiehlt in ihrer Fülle in be-sonderem Maße das Buch als Lehrbuch. Für die nächste Auflage sollten dann auch die histologischen Bilder und die bei Entzündungen ebenfalls in Farbe erscheinen. Es besteht kein Zweifel, daß diese fünfte Auflage weite Verbreitung finden wird und einer Generation von Zytologen und zytologisch ar-beitenden technischen Assistentinnen als Lehrbuch und Atlas dienen wird.

\section{A. Pfleiderer, Freiburg}

Gastrointestinale Malignome. Interdisziplinäre Therapie-konzepte

Herausgeber: H. Bünte, J. Meyer, P. Preusser Deutscher Ärzteverlag, Köln, 1990168 S., 70 Abb., 51 Tab., geb., DM 128-ISBN 3-7691-0215-0

Das Buch geht auf Erfahrungen einer interdisziplinären Stu-diengruppe in Münster zurück und orientiert sich an einem im Januar 1990 veranstalteten Symposium. Es wird zunächst generell festgestellt, daß die Prognose von Patienten mit ga-strointestinalen Malignomen nicht allein von der Therapie, sondern von verschiedenen Eingangsfaktoren wie Tumorsta-dium, Grading, Wachstumsfaktoren abhängt. Dementspre-chend werden die Ausführungen zu den verschiedenen Tumo-ren mit einem Beitrag zur Pathomorphologie und Biologie eingeleitet. Nacheinander werden das Ösophaguskarzinom, das Magenkarzinom, Pankreaskarzinom und die kolorektalen Karzinome abgehandelt, wobei jeweils ein Chirurg und ein in-ternistischer Onkologe Stellung nehmen. Die Strahlenthera-pie wird meist in den Kapiteln über die Chemotherapie mit abgehandelt.

Das Buch ist übersichtlich gegliedert. Hervorzuheben ist eine sehr gute und reichhaltige Auflockerung der Texte durch Tabellen, Grafiken und Abbildungen (32 Farbabbildungen). Die verschiedenen, vorwiegend mulitmodalen Therapie-ansätze werden übersichtlich dargestellt. Dennoch dürfte es dem reinen Praktiker schwerfallen, daraus plausible The-rapieansätze für 
seine Patienten abzuleiten. Insofern ist dieses Buch vorwiegend für den Fachonkologen geeignet, diesem vermag es jedoch sehr wertvolle Anregungen für seine praktische Tätigkeit und dem wissenschaftlich Arbei-tenden auch für die Konzeption neuer Therapieprotokolle zu vermitteln. W. Queißer, Mannheim

Bedeutung des omphalo-portalen Systems bei der Dïagnostik und Therapie der Metastasenleber kolorektaler Karzinome

Herausgeber: H. Strosche

Thieme, Stuttgart, 1990

91 S., 46 Abb., 25 Tab., Kart., DM 58-

ISBN 3-13-755401-2

Im Verlauf der letzten Dekade wurde die lokoregionäre Chemotherapie von Lebermetastasen bevorzugt kolorektaler Karzinome sehr intensiv untersucht. Diese lokoregionäre Chemotherapie hat den Vorteil, daß hohe Zytostatikakonzen-trationen an den Tumor herangeführt werden können und die systemische Toxizität gleichzeitig im Vergleich zur intravenö-sen Chemotherapie gering ist. Bisher wurden Zytostatika über implantierbare KatheterInfusionssysteme hauptsäch-lich über die Leberarterie perfundiert. In dem vorliegenden Buch wird die Perfusion der Zytostatika über die Nabelvene in die Pfortader vorgestellt. Hierzu waren umfangreiche Untersuchungen zur Anatomie und Topographie der Nabelvene und ihrer Beziehungen zum Pfortadersystem notwendig. Die Technik dieses Vorgehens wird ausführlich mit sehr anschaulichem Bildmaterial dargestellt.

Die Erfolgsbeurteilung der Behandlung von Lebermetastasen litt bisher an dem Fehlen geeigneter Untersuchungstech-niken zur Beurteilung der Metastasengröße. In der klinischexperimentellen Studie prüft der Autor ein computerdensi-tometrisches Konzept zur rechnergestützten Metastasenmes-sung. Dieses Modell ermöglicht eine quantitative Berech-nung der Einzelkompartimente vor und unter der Therapie. Damit wurde eine Forderung vieler Kliniker nach Optimie-rung der Meßverfahren zur Größenbestimmung von Metasta-sen erfüllt. Es ist zu wünschen, daß diese neue Methodik eine weite Verbreitung erfährt, um vielleicht in der Zukunft durch die Kom-bination dieser lokoregionären Chemotherapie mit einer sy-stemischen Chemotherapie die Spätergebnisse beim metasta-sierenden Kolonkarzinom zu verbessern. Das vorliegende Buch kann Chirurgen und internistischen Onkologen zur Lektüre empfohlen werden. P. Drings, Heidelberg 538

Buchbesprechunger

Radiopathology of Organs and Tissues

Herausgeber: E. Scherer, C. Streffer, K. R. Trott Springer, Heidelberg, 1991496 S., 156 Abb., 56 Tab., geb., DM 448-ISBN 3-540-19094-5

Incidental Carcinoma of the Prostate

Herausgeber: /. Altwein, P. Paul, W. Schneider Springer, Heidelberg, 1991276 S., 89 Abb., 105 Tab., geb., DM 118-ISBN 3-540-53225-0

Das vorliegende Buch gibt einen umfangreichen Überblick über den derzeitigen Stand der insbesondere durch strah-lentherapeutische Maßnahmen ausgelösten biochemischen Effekte, zellulären Reaktionen, Auswirkungen auf Gewebe und Organe sowíe insbesondere die hierdurch hervorgerufe-nen akuten und chronischen Strahlenfolgen. Das Buch ist hierzu in Kapitel über die verschiedenen Organsysteme ge-gliedert. In den jeweiligen Kapiteln findet der Leser urnfang- 
reiche Informationen von den grundlegenden Gewebereak-tionen bis zu Einzelangaben über verwendete Dosen und Ne-benwirkungshäufigkeiten. Die Texte werden hierbei durch umfangreiche Grafiken, Tabellen und ein gutes Bildmaterial aufgelockert. Das vorliegende Buch ist insbesondere für den klinisch tätigen Strahlentherapeuten außerordentlich interes-sant. Es sollte aus diesem Grund in jeder Klinik-Bibliothek stehen.

U. Busch, Göttingen

Der Begriff des «lncidental» Karzinoms der Prostata umfaßt Geschwulste, die sich im Ausmaß, im Wachstumsverhalten (Aggressivität) und ihrer Prognose sehr unterscheiden. Es handelt sich um klinisch nicht in Erscheinung tretende Tumo-ren, die bei der Behandlung aus einem anderen Anlaß uner-wartet angetroffen werden und diagnostisch wie auch thera-peutisch Konsequenzen nach sich ziehen. Im Gegensatz zum klinischen manifesten Karzinom findet sich die zur Diskussion stehende Geschwulstform im Zentrum oder auch im ventralen Teil der Prostata. Es kann bei nahezu jeder zehnten TUR mit einem entsprechenden Befund gerechnet werden. Zur Diskussion steht die Möglichkeit, die Geschwulst mittels Screening-Untersuchungen, die regelmäßige PSAAnalysen und transrektale Sonographien einschließen, frühzeitiger auf-zudecken und gegebenenfalls durch Bestimmung des DNA-Ploidie-Index zu differenzieren. Die Prognose der Erkran-kung steht und fällt mit dem Geschwulstvolumen; Das Tib

\section{Mitteihing}

der Vereinsgründung

der OAO

Oberrheinische Arbeitsgemein-schaft für Onkologie «OAO» (Groupe de Travail des Oncologues du Rhin Supérieur «GTOR») (Association for Oncology of the Upper Rhine «AOR»). Die Oberrheinische Arbeitsgemeinschaft für Onkologie (OAO) besteht aus den in der Region Oberrhein tätigen Onkologen. Die Arbeitsgemeinschaft besteht seit mehreren Jahren und hat das Ziel, die Information zwischen den einzelnen onkologisch tätigen Gruppen zu verbessern und auch gemeinsame klinische Forschungsprojekte durch-zuführen. Um die Zusammenarbeit weiter zu verbessern, wurde nun beschlossen, einen Verein mit dem Namen «Oberrheinische Arbeitsgemeinschaft für Onkologie (OAO)» («GTOR/AOR») zu gründen. Dieser Verein wurde nun am 1. Februar 1991 gegründet. Der Verein bezweckt unter anderem:

Die Förderung der Zusammenarbeit zwischen Onkologen aus der Schweiz, Frankreich und der Bundesrepublik Deutschland.

Die Planung und Durchführung gemeinsamer klinischer Forschungsprojekte.

Die Förderung der klinischen Fortbildung.

Die Durchführung jährlicher gemeinsamer Arbeitstagungen.

Wenn Sie sich durch die skizzierten Aufgabenstellungen angesprochen fühlen, möchten wir Sie gerne einladen, der OAO (GTOR/AOR) beizutreten. Es besteht ein bescheidener

Mitgliedsbeitrag von sFr. 20,-pro Jahr.

Kontaktadresse:

Dr. H. Denz

Dept. Innere Medizin

Abteilung für Onkologie

Petersgraben 4

CH-4031 Basel (Schweiz)

Buchbesprechungen

539 
(A2)-Karzinom ist hinsichtlich der Therapie mit T2-Tumoren vergleichbar. In Abhängigkeit vom Alter und nach Ausschluß pelviner Lymphknotenmetastasen wird die radikale Prosta-tektomie favorisiert. $\mathrm{Ob}$ auch der Tia (Ai)-Tumor entspre-chend behandelt werden soil, wird diskutiert. In dem vorliegenden Buch sind die Möglichkeiten der Dia-gnostik, die Biologie und PathoHistologie, der Stellenwert der Second-Look-TUR sowie weitere therapeutische Richtli-nien zu dieser Erkrankung sorgfältig herausgearbeitet. Es handelt sich dabei um eine übersichtlich gegliederte Zusam-menstellung von 41 Beiträgen verschiedener Autoren, die den derzeitigen Wissensstoff über das Incidental-Karzinom der Prostata lückenlos wiedergeben. Die einzelnen Kapitel sind inhaltlich und didaktisch sorgfältig verfaßt und zum Teil mit exzellenten Abbildungen illustriert. Pathologische Er-kenntnisse, umfangreiche diagnostische Möglichkeiten und therapeutische Notwendigkeiten finden sich in einer einmali-gen Form aktualisiert. Für den Studenten und den in Ausbil-dung befindlichen Kollegen ist das Buch angesichts der großen epidemiologischen Bedeutung der Erkrankung über-aus lesenswert. Dem fortgeschrittenen Urologen dient es dar-über hinaus als hilfreiches Nachschlagwerk; ein umfangrei-ches Sachverzeichnis ermöglicht einen schnellen Zugriff zu jeder gewünschten Information.

N. Jäger, Hildesheim Hämatologie und Onkologie. Ein Bildatlas

U. Vehling-Kaiser, R. Munker, A. Schalhorn, W. Wilmanns

Springer, Berlin, 1991

Ca. 160. S., 212 Abb., geb., DM 140-

ISBN 3-540-52611-0

Die Münchner Autoren haben mit ihrem kurzgefaßten Bildatlas Hämatologie und Onkologie eine Lücke gefüllt. In die-sem geben sie in kompakter Form makroskopische Abbildungen von typischen hämatologischen und onkologischen Krankheitsbildern wieder. Weiterhin werden spezielle Thera-piemaßnahmen in der Hämatologie und Onkologie bei der Plasmaphorese, der Knochenmarkstransplantation, der Transfusionsmedizin und der Hyperthermie dargestellt. Sichtbare Nebenwirkungen von Zytostatika werden anschau-lich dargelegt. Ein gut bebilderter Überblick über Techniken in der Hämatologie und Onkologie, wie die Knochenmarks-punktion, sowie die Implantation von Hickman-Katheter und venöser Portsysteme werden vorgestellt. Die Zielgruppen sind klinisch tätige und niedergelassene Me-diziner sowie Medizinstudenten und in der Pflege tätige Mit-arbeiter. Die Kombination von kurzen Textabschnitten und sehr gut ausgewählten technisch hochwertigen Abbildungen ist als sehr gelungen zu bezeichnen. Sie wird insbesondere Medizinstudenten und dem Pflegepersonal eine Zuordnung von typischen Symptomen und Nebenwirkungen der Chemo-therapie erleichtern. Für den ausgebildeten Hämatologen und Onkologen stellen die Textpassagen überwiegend Be-kanntes dar. Den Autoren ist es hervorragend gelungen, dem Leser die Blickdiagnose von Erkrankungen und Therapienebenwirkun-gen sowie einen Einblick in bestimmte Untersuchungstechni-ken nahezubringen. Das Buch kann wärmstens empfohlen werden für alle in der Onkologie Tätigen. H. H. Fiebig, Freiburg 